\title{
Mössbauer spectroscopy with a high velocity resolution: Advances in biomedical, pharmaceutical, cosmochemical and nanotechnological research
}

\author{
M.I. Oshtrakh ${ }^{\mathrm{a}, \mathrm{b}, *}$, V.A. Semionkin ${ }^{\mathrm{a}, \mathrm{b}}$ \\ a Department of Physical Techniques and Devices for Quality Control, Institute of Physics and Technology, Ural Federal University, Ekaterinburg 620002, Russian Federation \\ ${ }^{b}$ Department of Experimental Physics, Institute of Physics and Technology, Ural Federal University, Ekaterinburg 620002, Russian Federation
}

\section{A R T I C L E I N F O}

Article history:

Received 29 December 2011

Received in revised form 23 February 2012

Accepted 7 March 2012

\section{Keywords:}

Mössbauer spectroscopy with a high

velocity resolution

Biomedical research

Pharmaceutical analysis

Meteorites

Nanoparticles

\begin{abstract}
A B S T R A C T
The methodological principles of velocity resolution as additional characteristic of the quality of both Mössbauer spectrometer velocity driving system and Mössbauer spectrum were briefly considered. Significantly better quality of Mössbauer spectra measured with a high velocity resolution in comparison with those measured with a low velocity resolution was demonstrated. The main advances of recent studies of iron containing biomolecules, pharmaceutical products, meteorite samples and nanoparticles using Mössbauer spectroscopy with a high velocity resolution were considered and advantages of this technique were shown.
\end{abstract}

(ㄷ) 2012 Elsevier B.V. All rights reserved.

\section{Introduction}

Mössbauer spectroscopy is a unique technique which is widely used for study various molecular and biomolecular systems as well as various other objects and materials containing Mössbauer isotopes (for instance, ${ }^{57} \mathrm{Fe},{ }^{119} \mathrm{Sn},{ }^{121} \mathrm{Sb},{ }^{127} \mathrm{I},{ }^{197} \mathrm{Au}$, etc.). This technique is sensitive to nuclear hyperfine interactions and, therefore, provides very precise information about the electronic and magnetic state of the nuclei, chemical bonds, structure of local environment, etc. On the basis of Rudolph Mössbauer discovery of recoilless $\gamma$-ray emission and absorption in 1957, which is known as the "Mössbauer Effect" or $\gamma$-resonance, this spectroscopic method is realized mainly by relative movement of the Mössbauer radioactive source and absorber containing the same Mössbauer nuclei. Therefore, Mössbauer spectrum obtained in transmission geometry represents resonance absorption peaks determined by the nuclear hyperfine interactions in relation to Doppler modulation of the energy of $\gamma$-rays. The main features of the Mössbauer effect and principles of Mössbauer spectroscopy can be found in well known publications (see, for instance, [1-6]).

One of the main parts of Mössbauer spectrometer is the Doppler modulation system (velocity driving system) because it determines

\footnotetext{
* Corresponding author at: Department of Physical Techniques and Devices for Quality Control, Ural Federal University, Ekaterinburg 620002, Russian Federation. Tel.: +7912 2837337 .

E-mail address: oshtrakh@mail.utnet.ru (M.I. Oshtrakh).
}

the energy shift of $\gamma$-rays to reach resonance absorption. Therefore, the quality of Mössbauer spectrum depends on the quality of the Doppler modulation system. Extremely high sensitivity of nuclear transitions in the case of hyperfine interactions to the $\gamma$ rays energy (down to $10^{-9} \mathrm{eV}$ for ${ }^{57} \mathrm{Fe}$ ) limits the velocity error for the driving system. Therefore, in the present review we consider briefly the quality of Mössbauer spectrometers on the basis of velocity driving system and velocity resolution. Further we discuss the main advances of Mössbauer spectroscopy with a high velocity resolution in biomedical, pharmaceutical, cosmochemical and nanotechnological research.

\section{Velocity resolution in Mössbauer spectroscopy}

The term "velocity resolution" is the term that suggested to apply for evaluation and characterization of the quality of both velocity driving system in Mössbauer spectrometer and measured Mössbauer spectrum in addition to differential and integral nonlinearity of the velocity reference signal, differential and integral nonlinearity of the velocity signal, time and temperature instability of the velocity signal amplitude, drift of the zero velocity point, noise of velocity signal, signal-to-noise ratio in the spectrum and so on. A simplified view of velocity reference signal formation in the constant acceleration mode by the $n$-ordered digital-analog converter as electrical signal with a set of $2^{n}$ steps is shown in the Scheme in Fig. 1. Velocity resolution in velocity driving system of Mössbauer spectrometer denoted the smallest velocity step $2 \mathrm{~V} / 2^{n}$, where $2 V$ is velocity range (from $-\mathrm{V}$ till $+\mathrm{V}$ ) and $n$ is the order of 


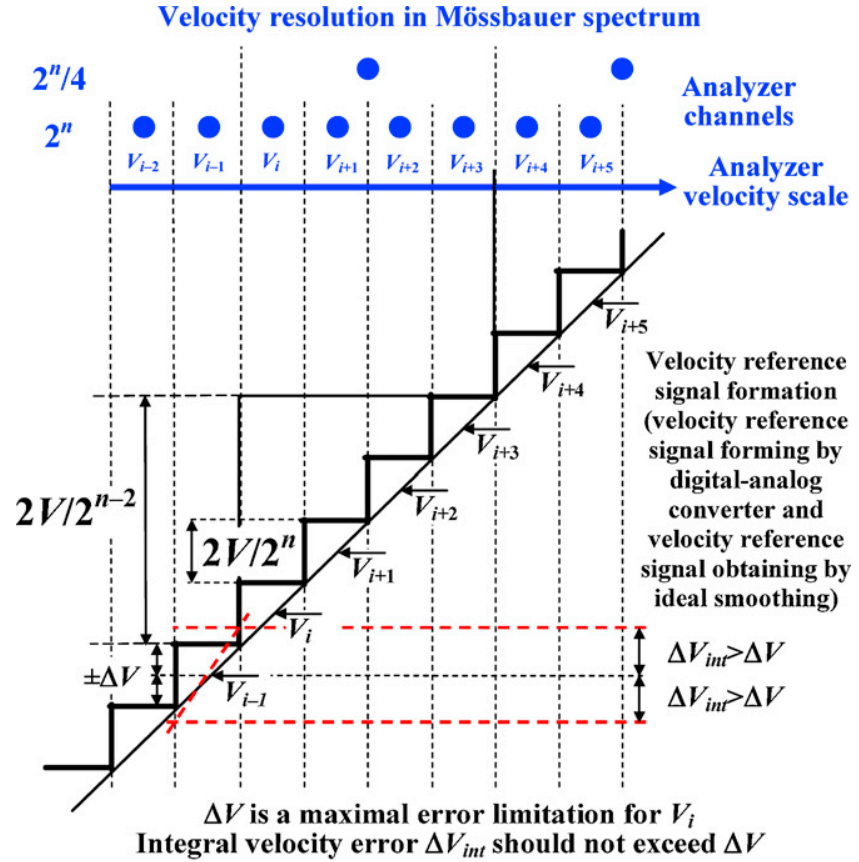

Velocity resolution in spectrometer driving system

Fig. 1. The scheme of the basis of velocity resolution in velocity driving system of Mössbauer spectrometer and in measured Mössbauer spectrum. Velocity reference signal is forming by digital-analog converter of the order $n$ as a set of $2^{n}$ steps. The velocity resolution is determined by $2 V / 2^{n}$ where $\pm V$ is the velocity range. The velocity resolution in the spectrum may be the same if $2^{n}$ channels of multichannel analyzer are used for the spectrum storage (each velocity interval $V_{i}$ will correspond, synchronized in time window, to the channel $i$ ). If integral error $\Delta V$ for velocity step $i$ exceeds $1 / 2$ of velocity step, the velocity resolution should be reduced by factor 2 , 4 , etc. In this case velocity resolution in the spectrum should be decreased by the same factor at least.

digital-analog converter. The limitation in increasing a velocity resolution is determined by the $n$ value with requirement that the integral error of the velocity step did not exceed a half of the velocity step. If this error exceed a half of the velocity step, it means that Doppler energy shift for $\gamma$-rays will overlap with that of the next or previous velocity steps and so on (see Scheme in Fig. 1). In this case smoothing of Mössbauer spectrum may be a result of the error. Velocity resolution in Mössbauer spectrum is velocity step per one spectrum point (one channel of multichannel analyzer). Velocity resolution coefficient $1 / 2^{n}$ in the velocity driving system in the chosen spectrometer is constant and velocity resolution value depends on velocity range $(2 \mathrm{~V})$ only while velocity resolution in Mössbauer spectrum may be the same or less (for instance, $2^{n-1}, 2^{n-2}$, and so on) due to decrease of channel number in multichannel analyzer.

After formation of the velocity reference signal its smoothing take place resulting in velocity signal close to linear (the ideal signal has linear or sinusoidal shape). This signal is transferred into the system of mechanical movement for Doppler modulation of the resonance $\gamma$-rays. During the digital-analog converter "time window" (the time for each velocity step $t_{i}$ ) there is a change of smoothed velocity from $V_{i 1}$ till $V_{i 2}$ (see Scheme in Fig. 2). Thus, there is a Doppler modulation of the $\gamma$-ray energy in the range of $E_{\gamma}\left(1 \pm V_{i 1} / c\right) \div E_{\gamma}\left(1 \pm V_{i 2} / c\right)$ during this time. The main requirement of stability of the Doppler modulation system of Mössbauer spectrometer is the constant value of $2 V / 2^{n}$ (and, therefore, the value of $\left.V_{i 2}-V_{i 1}\right)$ and the "time window" $t_{i}$. In this case there is a correspondent Doppler modulation of the energy of $\gamma$-rays for each $i$-th range of velocity change. The registration of these $\gamma$-rays depends on condition of multichannel analyzer discretization. If the number of velocity steps and the number of channels of multichannel
Velocity resolution in Mössbauer spectrum $\pm \Delta V$ is a systematic velocity error for each channel

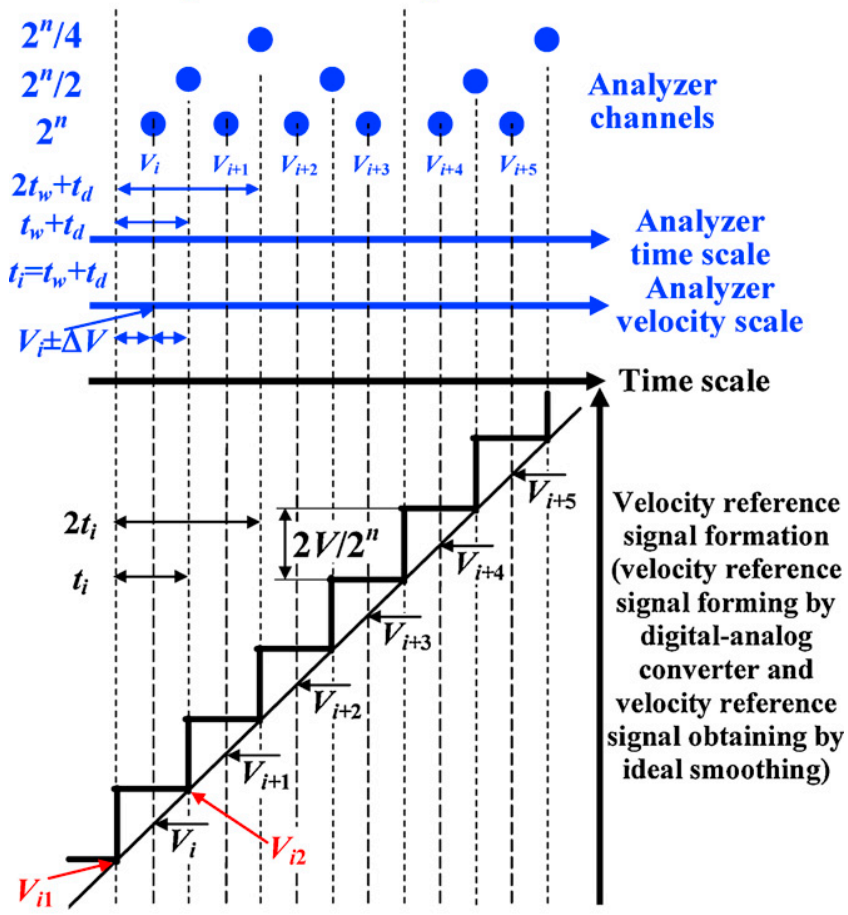

Velocity resolution in spectrometer driving system

Fig. 2. The scheme of the reason of instrumental (systematic) error for each spectrum point on velocity scale in the case of constant acceleration mode. The "time window" for the $i$-th velocity step of the digital-analog converter is $t_{i}, V_{i}$ is a velocity corresponding to $1 / 2 t_{i}, V_{i 1}$ and $V_{i 2}$ are the low and upper limits of the $i$-th velocity range of the smoothed velocity reference signal, $t_{\mathrm{w}}$ is a time window for each of $2^{n}$ channels of multichannel analyzer, $t_{\mathrm{d}}$ is a dead time for multichannel analyzer.

analyzer are the same $\left(2^{n}\right)$, there is consequent registration of $\gamma$ rays with Doppler modulation of the $i$-th velocity range into the $i$-th channel of the analyzer memory. The sum of the time window of the analyzer memory channel $\left(t_{\mathrm{w}}\right)$ and the analyzer dead time $\left(t_{\mathrm{d}}\right)$, i.e. the time of memory channels switching, are equal to $t_{i}$. In this case the $i$-th channel will be assigned the velocity value of the $i$-th velocity range $V_{i}$ which is correspondent to $1 / 2 t_{i}$. The integral error of the velocity signal for the $i$-th velocity range should not exceed $\pm\left(2 V / 2^{n}\right) / 2$ with respect to $V_{i}$, otherwise the Doppler modulation of the $\gamma$-rays energy will exceed the range of $E_{\gamma}\left(1 \pm V_{i 1} / c\right) \div E_{\gamma}\left(1 \pm V_{i 2} / c\right)$ during the time $t_{i}$. In the latter case of the $\gamma$-rays with the Doppler modulation energy correspondent to the neighboring velocity ranges $(i-1$ и $i+1)$ would be registered in the $i$-th channel. Therefore, it requires to decrease the velocity resolution in the Doppler modulation system until the integral velocity error will be less than $\pm\left(2 V / 2^{n}\right) / 2$ with respect to $V_{i}$. The value of $\pm\left(2 V / 2^{n}\right) / 2$ is a constant for all velocity ranges $\left(i=1 \div 2^{n}\right)$. Therefore, it can be used as the maximal instrumental error for chosen velocity resolution in the Doppler modulation system. As for the instrumental error for the velocity value $V_{i}$ for the $i$-th channel of analyzer memory, it will be determined by the value of $\pm\left(2 \mathrm{~V} / 2^{n}\right) / 2$ also. This value in fact characterizes the limit of velocity variation in the $i$-th velocity range which is correspondent to the $i$-th channel of analyzer and can be considered as the instrumental (systematic) error for each spectrum point on the velocity scale. It should be noted that, in contrast, in the case of constant velocity mode in the driving system the error of velocity $V_{i}$ for the $i$-th channel of analyzer memory is determined by the error of constant velocity $V_{i}$ formed by the driving system only. Therefore, an increase in 
velocity resolution leads to decrease in the instrumental (systematic) error for the velocity scale.

Mössbauer spectroscopy with a high velocity resolution is a new method to measure precision high quality spectra. Usual spectrometers are used sinusoidal or triangular velocity reference signal and 256 or 512 bits to form velocity signal on both direct and reverse motion. Such velocity driving system provides spectra measurement with a low velocity resolution (in $2^{n}=256$ or 512 channels) with possibility to decrease measurement time and reach needed signal-to-noise ratio by spectra folding on the direct and reverse motion. However, these driving systems do not provide a low instrumental (systematic) error for velocity signal while folding increases integral velocity error due to different velocity errors on the direct and reverse motions. These problems can be neglected if a high precision is not required for spectra measurement. Nevertheless, further development of Mössbauer spectroscopy may be related to increase in precision and quality of spectra measurement with less instrumental (systematic) velocity error and to increase in velocity resolution for both spectrometer and spectrum. Earlier we have pointed out that the high quality and precise Mössbauer spectrometers with a high velocity resolution are needed for further development of biomedical application of Mössbauer spectroscopy [7]. Moreover, application of this technique in other fields of research may be also useful because in this case it is possible to decrease the instrumental (systematic) error for each spectrum point in the velocity scale and to reach more reliable fit of complicated spectra due to increase in the number of spectra points.

\section{Experimental technique and some comparison}

Mössbauer spectra of various samples were measured using an automated precision Mössbauer spectrometric system created on the basis of the spectrometer SM-2201 with saw-tooth shape velocity reference signal and a high velocity resolution ( 4096 bits to form velocity reference signal), and temperature variable liquid nitrogen cryostat with moving absorber. The noise of velocity signal of the spectrometer was $1.5 \times 10^{-3} \mathrm{~mm} / \mathrm{s}$, the drift of zero velocity was $\pm 2.6 \times 10^{-3} \mathrm{~mm} / \mathrm{s}$, the nonlinearity of velocity signal was $0.01 \%$, the systematic error of velocity setting was $0.025 \%$ and the temperature drift of the velocity signal was $2.6 \times 10^{-6}(\mathrm{~mm} / \mathrm{s}) /{ }^{\circ} \mathrm{C}$. These values were obtained by spectra measurements of $\alpha$-Fe standard absorber in a large velocity range using 4096 channels. In the case of velocity range decrease down to $\pm 2 \mathrm{~mm} / \mathrm{s}$, values of the noise of velocity signal of the spectrometer, the drift of zero velocity and the temperature drift of the velocity signal respectively decreased and did not exceed a half of channel value in $\mathrm{mm} / \mathrm{s}$. Details of this equipment and the system were given elsewhere [8,9]. The Mössbauer spectra were measured in transmission geometry with moving absorber in the cryostat at a temperature range $295-90 \mathrm{~K}$ and recorded in 4096 channels. For their analysis, spectra with a low iron content and poor signal-to-noise ratio were converted into 1024 channels by a consequent summation of four neighboring channels while spectra with a good signal-to-noise ratio were converted into 2048 channels by a consequent summation of two neighboring channels or used as measured (in 4096 channels). Statistical counting rate in the spectra varied from $0.5 \times 10^{6}$ to $26.0 \times 10^{6}$ counts per channel. The measurement time varied from about $24 \mathrm{~h}$ till 60 days. The signal-to-noise ratio for obtained spectra was in the range between $\sim 12$ and $\sim 140$. These values were dependent on the iron content in the samples. Biological samples had effective thickness varied from 0.1 till $0.9 \mathrm{mg} \mathrm{Fe} / \mathrm{cm}^{2}$ while other samples (pharmaceutical samples, purified ferritin, meteorite samples, nanoparticle samples) had effective thickness in the range from 5 till $12 \mathrm{mg} \mathrm{Fe} / \mathrm{cm}^{2}$. The standard absorbers of $\alpha$-Fe foil with a thickness of $10 \mu \mathrm{m}$ and sodium nitroprusside with effective thickness of $10 \mathrm{mg} \mathrm{Fe} / \mathrm{cm}^{2}$ were used for calibration of the velocity scale in large and low velocity ranges, respectively. The spectra were computer fitted with the least squares procedure using UNIVEM-MS program with Lorentzian line shape. It should be noted that there were no thickness effect in the spectra of studied samples with a large amount of iron. Therefore, there was no need for a transmission integral method to be used for the spectra fitting. Spectral parameters such as: isomer shift, $\delta$, quadrupole splitting, $\Delta E_{\mathrm{Q}}$, line width, $\Gamma$, relative subspectrum area, $S$, and statistical quality of the fit, $\chi^{2}$, were determined. The instrumental (systematic) error for each spectra point on the velocity scale was \pm 0.5 channel, the instrumental (systematic) error for hyperfine parameters evaluation was \pm 1 channel while the instrumental (systematic) error for $\Gamma$ evaluation was \pm 2 channels. It should be noted that spectrometer characteristics determined an integral velocity error (total mechanical and electronics systematic and random errors) which was several times less than a half of channel value in $\mathrm{mm} / \mathrm{s}$ during spectra measurements using 4096 channels [8]. If error calculated with the fitting procedure (fitting error) for these parameters exceeded the instrumental (systematic) error, we used the larger error. Velocity resolution (velocity per one channel) was $2 \mathrm{~V} / 2^{n} \mathrm{~mm} / \mathrm{s}$ per channel for $2^{n}$ channels ( $n$ in the spectra varied from 10 to 12 , velocity range $V$ varied from \pm 2.5 till $\pm 12.0 \mathrm{~mm} / \mathrm{s}$ ). The relative error for $S$ did not exceed $10 \%$. Criteria of the best fit were differential spectra, $\chi^{2}$ values and physical meaning of parameters. The values of standard deviation $(\sigma)$ of $\chi^{2}$ were $0.044,0.033$ and 0.022 for spectra presented in 1024 , 2048 and 4096 channels, respectively. Values of $\delta$ are given relative to $\alpha$-Fe at $295 \mathrm{~K}$.

Measurements of the standard absorbers using three Wissel spectrometers, one KFKI and one MsAa-3 spectrometers with triangular velocity reference signal were done at the other laboratories for evaluation of the spectra quality using $\alpha$-Fe (in some cases we used our own standard absorber of $\alpha$-Fe foil with a thickness of $10 \mu \mathrm{m}$ ). These spectra were fitted using UNIVEM-MS program. Some of these results are presented further in comparison with similar measurements using our Mössbauer spectrometric system. Mössbauer spectrum of $\alpha$-Fe measured using Wissel spectrometer with a low velocity resolution (in 252 channels) is shown in Fig. 3. The full spectrum measured on both direct and reverse motion demonstrated inverse parabolic distortion (Fig. 3a) which should be excluded after folding. The difference between two spectra measured on the direct and reverse motion demonstrated that there are different velocity errors for two motions (Fig. 3b) which increased the error for each spectrum point after folding and may change the line shape. It should be noted that this difference was more or less observed for all spectrometers with a low velocity resolution used in this comparison. In some spectrometers parabolic distortion of the base spectrum line remained after folding that again indicated some differences in velocity signal and its error on the direct and reverse motion. The results of the fit of the Mössbauer spectra measured on the direct motion and obtained after folding with Lorentzian line shape are shown in Fig. $3 \mathrm{c}$ and d. It is clearly seen that these spectra were not fitted well (see differential spectra). The values of $\chi^{2}$ were 37.8 and 59.7, respectively, that indicated different quality of both spectra fit. Moreover, it was interesting to observe that in the case of the fit using Lorentzian-Gaussian line shape, about $40 \%$ of Gaussian line was obtained in the fit with decrease of $\chi^{2}$ values: $\chi^{2}=25.7$ and $\chi^{2}=42.0$ for the direct motion and folded spectra, respectively. The instrumental (systematic) errors for these spectral points were $\sim 0.2 \mathrm{~mm} / \mathrm{s}$ for direct motion and $\sim 0.4 \mathrm{~mm} / \mathrm{s}$ after folding (for 252 channels). Similar results were obtained for the fit of other spectra measured using Wissel and KFKI spectrometers in 512 and 256 channels, respectively. It should be noted that non-Lorentzian line shape may also be a result of the thickness effect. However, in the case of measurement of our 

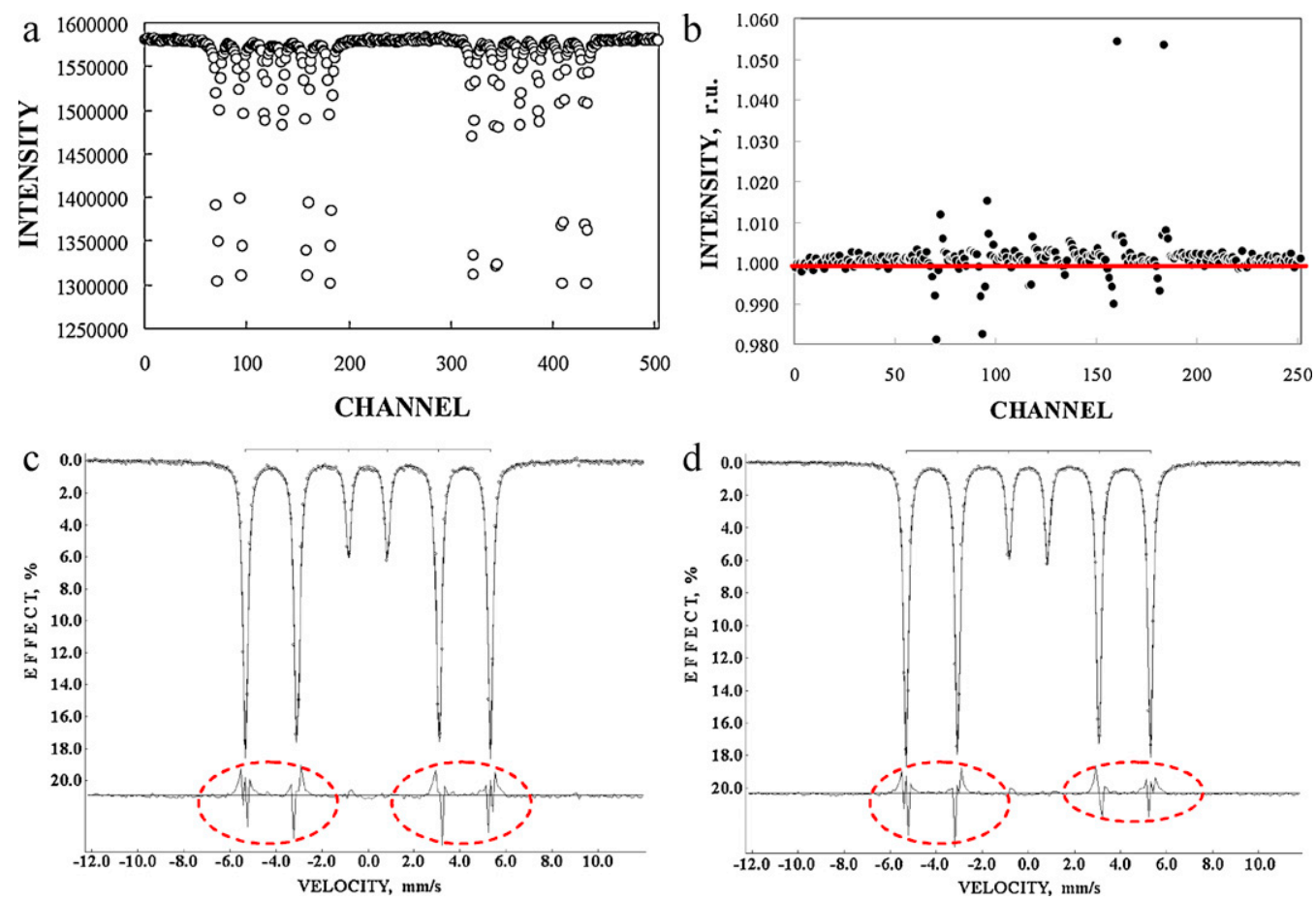

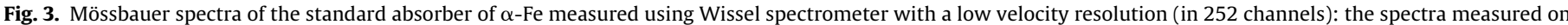
the direct and reverse motion (a), the difference between the direct and reverse motion spectra (b), the direct motion spectrum (c), and the spectrum after folding (d).

standard absorber using KFKI spectrometer, the absorption line shape remained non-Lorentzian also with less part of the Gaussian line shape (about 10-15\%). In contrast, MsAa-3 spectrometer [10] with a high velocity resolution (4096 bits to form triangular velocity reference signal) demonstrated much better quality of the spectrum. The line shape was Lorentzian without Gaussian admixture like in Wissel and KFKI spectrometers but with residual parabolic distortion after folding and twice larger instrumental (systematic) error for each spectrum point due to spectrum folding. Mössbauer spectra of the standard absorbers measured using our Mössbauer spectrometric system with a high velocity resolution (in 4096 channels) are shown in Fig. 4. These spectra were fitted using Lorentzian line shape with $\Gamma_{1,6}=0.250 \mathrm{~mm} / \mathrm{s}$, $\Gamma_{2,5}=0.243 \mathrm{~mm} / \mathrm{s}, \Gamma_{3,4}=0.233 \mathrm{~mm} / \mathrm{s}( \pm 0.008 \mathrm{~mm} / \mathrm{s})$ for $\alpha$-Fe and $\Gamma=0.238 \pm 0.003 \mathrm{~mm} / \mathrm{s}$ for sodium nitroprusside. It was also shown the absence of Gaussian shape admixture. Thus, comparison carried out demonstrated better quality of Mössbauer spectra measured with a high velocity resolution.

\section{Advances of Mössbauer spectroscopy with a high velocity resolution}

\subsection{Biomedical application}

Biomedical applications require a very careful measurement of Mössbauer spectra especially in the case of comparison of normal and pathological subjects. The first results of the application of Mössbauer spectroscopy with a high velocity resolution in biomedical research were recently considered in [11-16]. One of the main results obtained in the study of iron storage protein ferritin extracted from human liver demonstrated that an increase in statistical counting rate in the spectrum cannot compensate an increase in velocity resolution (in the number of spectral points) [17]. Mössbauer spectra of ferritin presented with different velocity resolution from 4096 down to 256 channels after measurement in 4096 channels with corresponding increase in statistical counting rates (up to 16 times for 256 channels spectrum) were fitted
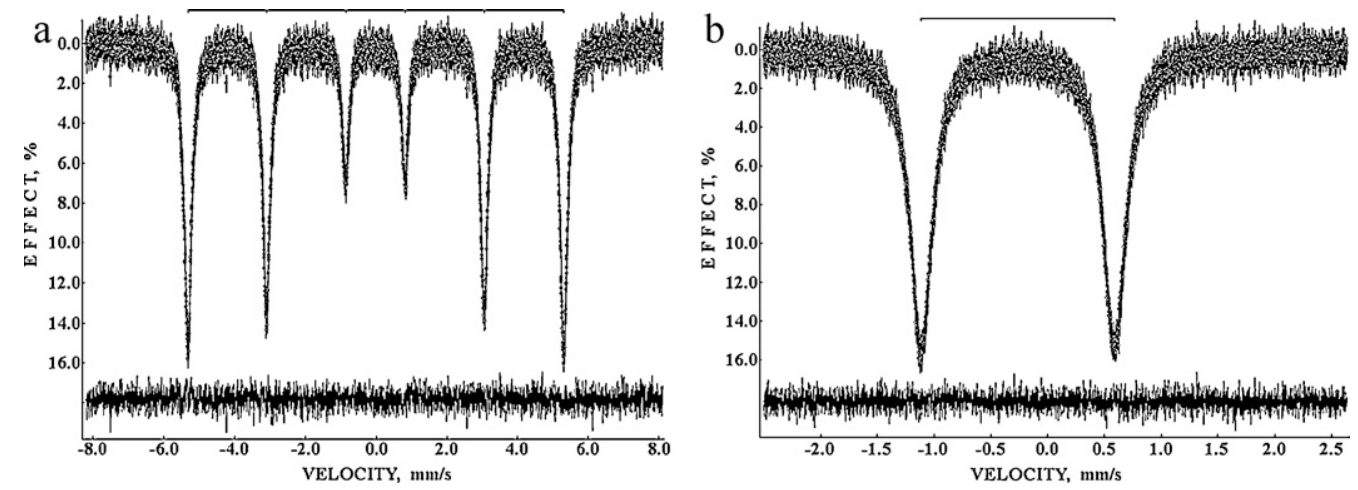

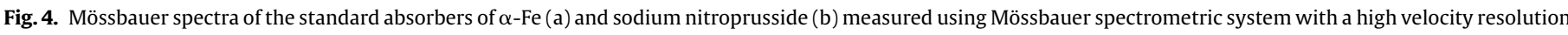
in 4096 channels at room temperature. 

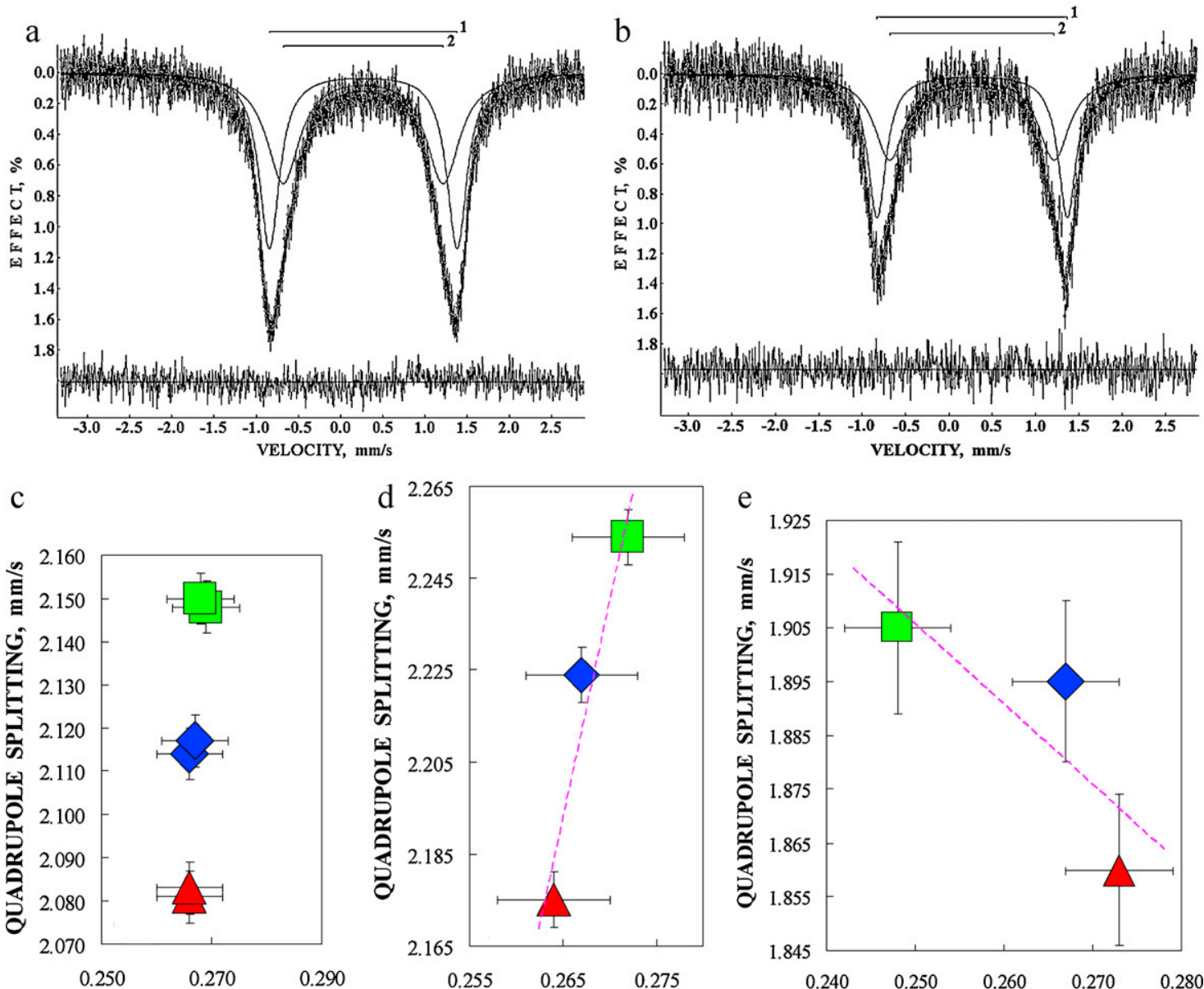

ISOMER SHIFT, $\mathrm{mm} / \mathrm{s}$

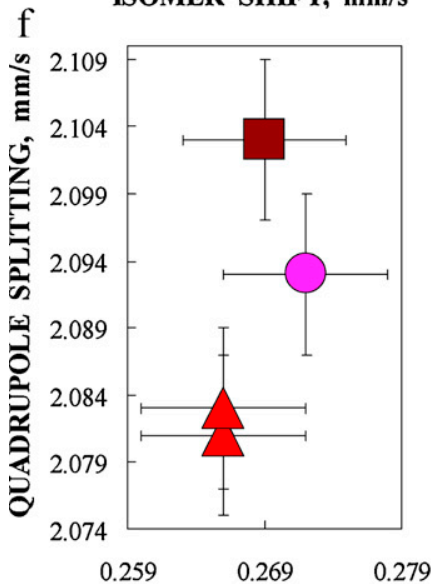

ISOMER SHIFT, $\mathrm{mm} / \mathrm{s}$

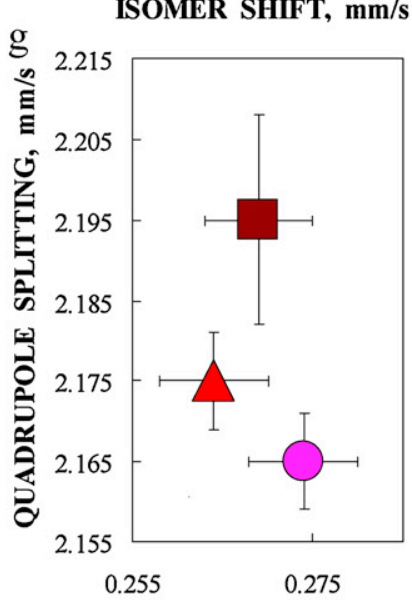

ISOMER SHIFT, $\mathrm{mm} / \mathrm{s}$ $\mathrm{e}$

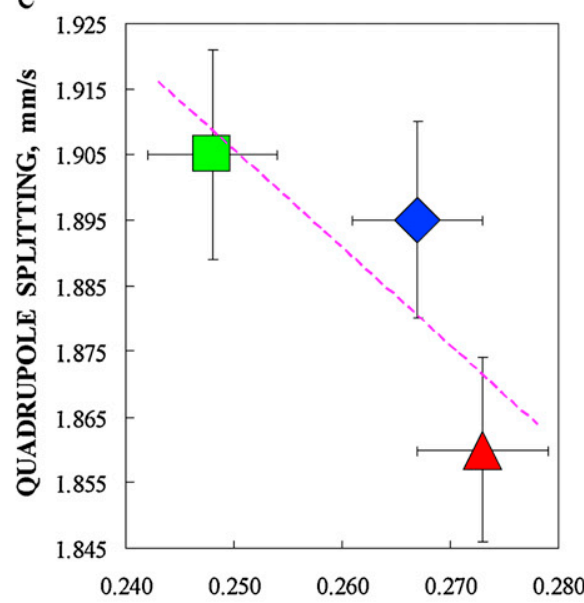

ISOMER SHIFT, $\mathrm{mm} / \mathrm{s}$

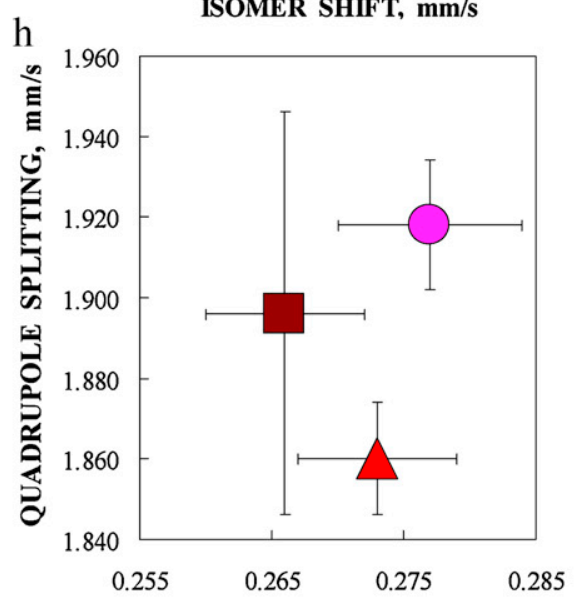

ISOMER SHIFT, $\mathrm{mm} / \mathrm{s}$

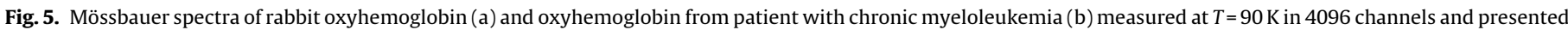

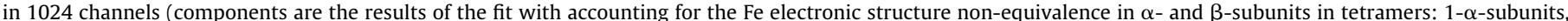

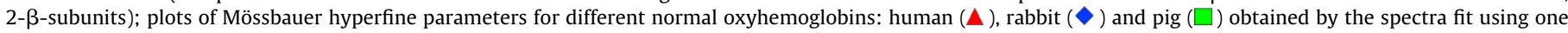

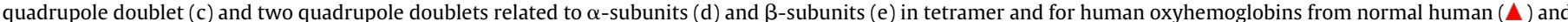

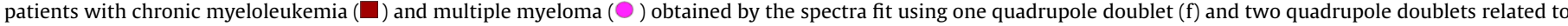
$\alpha$-subunits (g) and $\beta$-subunits (h).

with the same model of four quadrupole doublets. However, the results of these fits were different, especially, between the low and high velocity resolution spectra. Therefore, results of the fit of Mössbauer spectra with a high velocity resolution even within the same model were more reliable in spite of lower statistical counting rates. Moreover, a decrease in the instrumental (systematic) error for each spectrum point and Mössbauer hyperfine parameters with increase in velocity resolution demonstrated the possibility to distinguish small variations of quadrupole splitting and isomer shift (in the case when the fitting error was less then systematic one).

Further results were obtained in the study of normal oxyhemoglobin in red blood cells from normal adult human, rabbit and pig as well as oxyhemoglobin in red blood cells from patients with 

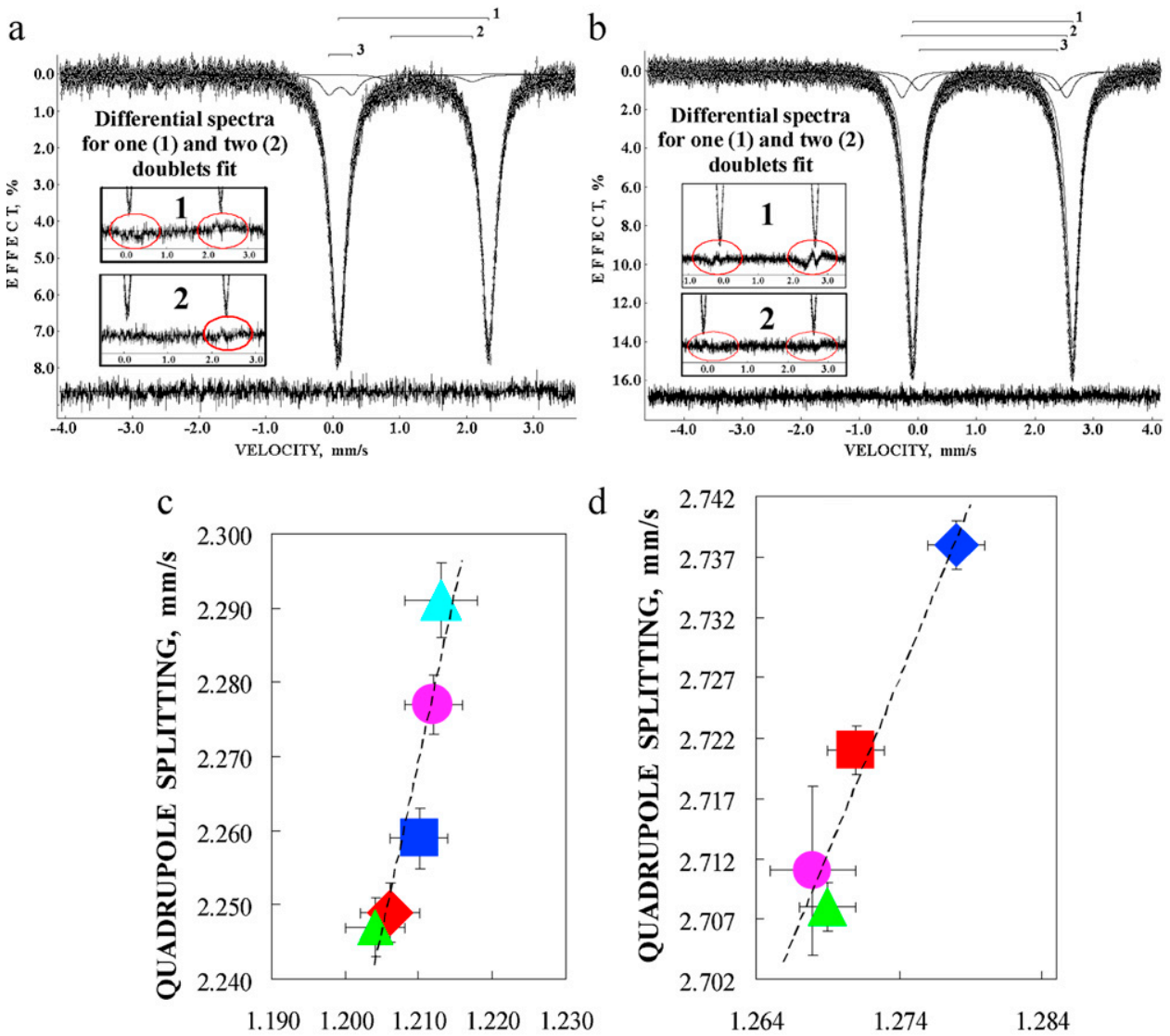

ISOMER SHIFT, $\mathrm{mm} / \mathrm{s}$

ISOMER SHIFT, $\mathrm{mm} / \mathrm{s}$

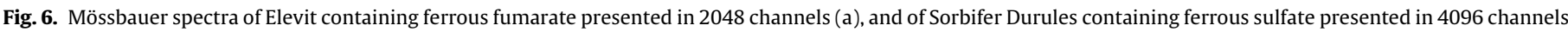

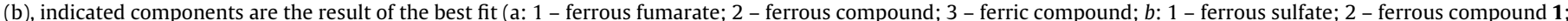

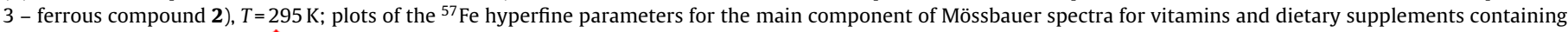

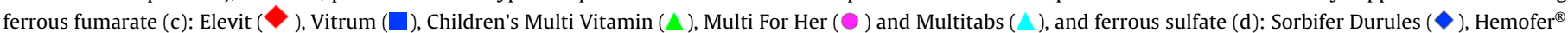
$(\square)$, Falvit ${ }^{\circledR}(\triangle)$ and Vitaral $(\bigcirc)$.

chronic myeloleukemia and multiple myeloma [18-20]. Mössbauer spectra were measured at $90 \mathrm{~K}$ in 4096 channels and then converted into 1024 channels due to a low iron content (see spectra of rabbit oxyhemoglobin and oxyhemoglobin from patient with chronic myeloleukemia in Fig. 5a and b). These spectra demonstrated asymmetry of the absorption line shape and, therefore, were fitted using two models: one quadrupole doublet (equivalence of the $\mathrm{Fe}(\mathrm{II})$ electronic structure in $\alpha$ - and $\beta$-subunits of tetrameric oxyhemoglobin) and two quadrupole doublets with the same area (non-equivalence of the $\mathrm{Fe}(\mathrm{II})$ electronic structure in $\alpha$ - and $\beta$ subunits of tetrameric oxyhemoglobin). The first model should be considered as rough approximation. We used this model taking into account the fact that majority of Mössbauer spectra of oxyhemoglobin measured in previous years by other researches were fitted in this way. However, the second model may reflect well known structural differences in the heme Fe(II) environment in $\alpha$ and $\beta$-subunits of tetrameric oxyhemoglobin [21]. The results of the fit using the first model for normal oxyhemoglobins demonstrated the possibility to distinguish the hyperfine parameters for human, rabbit and pig oxyhemoglobins beyond the instrumental (systematic) error (Fig. 5c). Application of the second model demonstrated two different quadrupole doublets with different intensities and line widths. This was related to different oxygen molecule orientation in the heme cavity of the $\alpha$ - and $\beta$-subunits (see [22]). The obtained values of the hyperfine parameters were related to the $\alpha$-subunits (the doublet 1 ) and to the $\beta$-subunits (the doublet 2). These values for three normal oxyhemoglobins were distinguished beyond the error also (Fig. $5 \mathrm{~d}$ and e). Linear variation of the Mössbauer hyperfine parameters indicates some small changes in the $\sigma$-and $\pi$-bonds of the heme Fe(II) for both subunits in different oxyhemoglobins. The differences obtained correlated with small structural variations for the heme Fe(II) stereochemistry in three different hemoglobins as well as in their $\alpha$ - and $\beta$-subunits. Moreover, it was found a correlation of quadrupole splitting and hemoglobins' oxygen affinity. Similar but smaller differences were obtained in the fits of patients' oxyhemoglobins using two models (Fig. $5 \mathrm{f}-\mathrm{h}$ ). It should be noted that these differences of hyperfine parameters would not be possible to observe in Mössbauer spectra measured with a low velocity resolution in the case of indication of correct instrumental (systematic) error.

Recent comparative study of chicken liver obtained from chicken with two different ages and from two different poultry farms using Mössbauer spectroscopy with a high velocity resolution demonstrated small variations of the hyperfine parameters in spite of extremely low iron content and poor signal-to-noise ratio [23]. This fact may be a result of differences in the ferritin iron cores related to the chicken age and feeding.

\subsection{Pharmaceutical application}

A number of pharmaceutical compounds contains Mössbauer atoms such as Fe, Sn, Au. Pharmaceuticals containing Fe are mainly used for iron deficiency treatment. Some of them are models of ferritin and consist of ferric hydrous oxide nanosized core in 


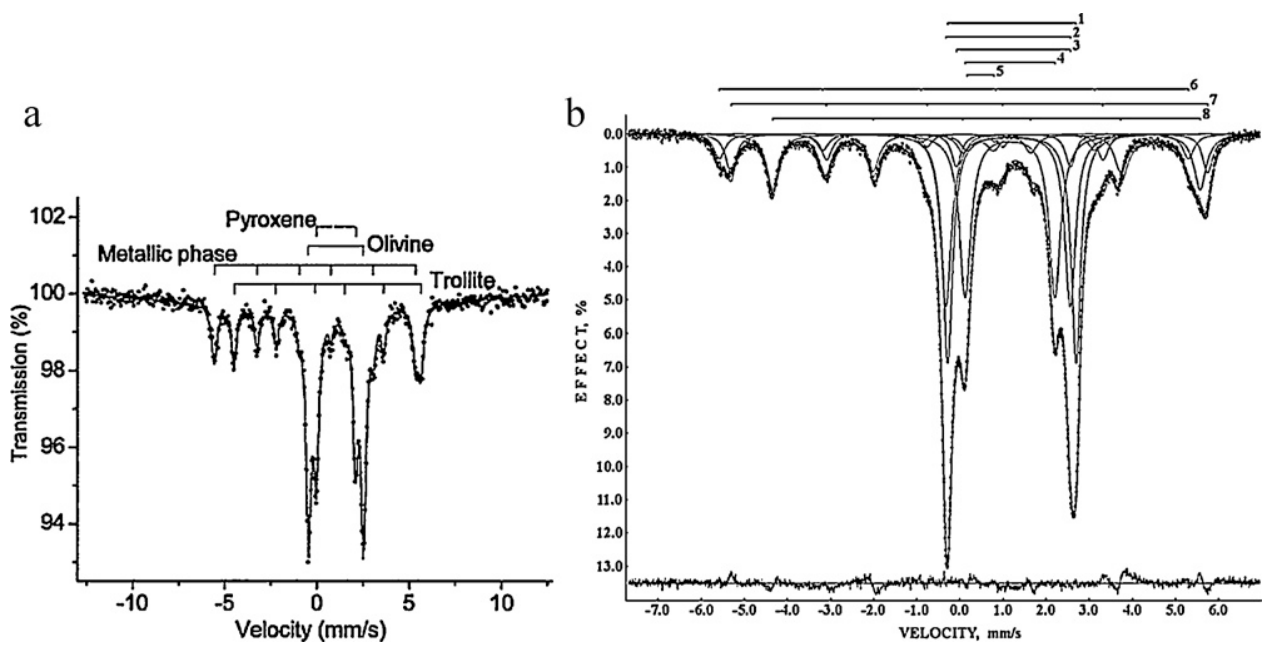

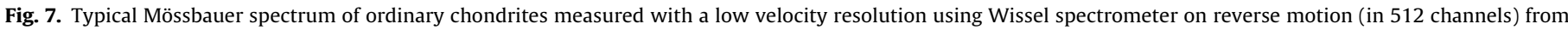

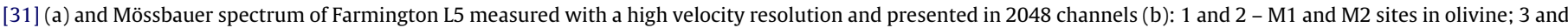
$4-\mathrm{M} 1$ and M2 sites in pyroxene; $5-\mathrm{Fe}^{3+} ; 6$ and 7 - metallic phase; 8 - troilite; $T=295 \mathrm{~K}$.

the form of $\beta-\mathrm{FeOOH}$ surrounded with polysaccharides (dextran, dextrin, polymaltose, etc.). The study of commercially distributed medicines Imferon, Maltofer ${ }^{\circledR}$ and Ferrum Lek using Mössbauer spectroscopy with a high velocity resolution demonstrated some differences which could not be distinguished using Mössbauer spectroscopy with a low velocity resolution $[23,24]$. Based on these results a new model for the iron cores in ferritin and its models was suggested.

Various vitamins and dietary supplements contain Fe in the form of ferrous fumarate and ferrous sulfate. Recently several commercial samples of Elevit (Rottendorf Pharma GmbH, Germany), Vitrum (Unifarm, Inc., USA), Children's Multi Vitamin (Target Corporation Minneapolis, USA), Multi For Her (Nature Made Nutritional Products, USA) and Multitabs (Ferrosan A/S, Denmark) containing the ferrous fumarate, and Sorbifer Durules (Egis, Hungary), Hemofer ${ }^{\circledR}$ (Glaxo-SmithKline Medicines S.A., Poland), Falvit ${ }^{\circledR}$ (Jelfa, Poland) and Vitaral (Jelfa, Poland) containing the ferrous sulfate were studied using Mössbauer spectroscopy with a high velocity resolution $[25,26]$. Mössbauer spectra of Elevit and Sorbifer Durules are shown in Fig. 6a and b. These spectra were fitted using different models (numbers of components). Visually, the high velocity resolution Mössbauer spectra of these and other measured spectra look like single quadrupole doublet however they were better fitted using several quadrupole doublets. It was interesting to observe two minor ferrous and ferric components in the spectra of Elevit, Vitrum and Multitabs, and one minor ferrous component in the spectra of Children's Multi Vitamin and Multi For Her. On the other hand, two minor ferrous components 1 and 2 were observed in the spectra of Sorbifer Durules, Hemofer ${ }^{\circledR}$ and Vitaral as well as two minor ferrous and one ferric components were observed in the spectrum of Falvit ${ }^{\circledR}$. These minor components may be considered as impurities. It should be noticed that the relative areas of the ferric compounds found from the spectra of Elevit and Vitrum were equal to $\sim 5.0 \%$ and $\sim 3.7 \%$, respectively. These relative amounts of the ferric impurities exceed FDA allowed limit for the ferrous fumarate drugs which should contain no more than $2 \%$ of the ferric iron (Food and Drugs, Sec. 172.350). The ${ }^{57}$ Fe hyperfine parameters $\delta$ and $\Delta E_{\mathrm{Q}}$ obtained for the main spectral components from the best fits of all Mössbauer spectra of the studied samples are shown in Fig. $6 \mathrm{c}$ and $\mathrm{d}$. It is interesting to see that in two groups of pharmaceutical products containing both ferrous fumarate and ferrous sulfate the hyperfine parameters of the main components appeared to be slightly different beyond the instrumental (systematic) error within a corresponding group. These small variations of the ${ }^{57} \mathrm{Fe}$ hyperfine parameters for the ferrous fumarates and the ferrous sulfates were revealed thanks to a high velocity resolution of the used equipment and, hence, to a significant decrease in the instrumental (systematic) error of the spectral parameters. This fact may indicate that the ferrous fumarate in different vitamins and dietary supplements as well as the ferrous sulfate in various studied samples were slightly different as a result both of various processes of vitamins production applied by their manufacturers, use of different components and/or similar components but of a different origin.

\subsection{Cosmochemical application}

Various meteorites contain Fe and, therefore, were being studied using Mössbauer spectroscopy for a long time. However, investigations of some meteorites samples using Mössbauer spectroscopy with a high velocity resolution demonstrated new results in revealing of spectral components which cannot be obtained from the low velocity resolution Mössbauer spectra [12,27-30]. For instance, in the study of ordinary chondrites it was possible to fit complicated Mössbauer spectra using components related to the crystallographically non-equivalent $\mathrm{M} 1$ and $\mathrm{M} 2$ sites occupied by both $\mathrm{Fe}^{2+}$ and $\mathrm{Mg}^{2+}$ ions in both pyroxene $(\mathrm{Fe}, \mathrm{Mg}) \mathrm{SiO}_{3}$ and olivine $(\mathrm{Fe}, \mathrm{Mg})_{2} \mathrm{SiO}_{4}$ while in the low velocity resolution spectra this was not possible (see comparison of Mössbauer spectra of ordinary chondrites measured in [31] with a low velocity resolution and with a high velocity resolution (this work) in Fig. 7). Moreover, it was also possible to observe several components related to metallic phase in the case of complicated Fe-Ni alloy structure. Further studies of meteorites and some compounds extracted from meteorites demonstrated new interesting results. Mössbauer study of olivine extracted from pallasites Omolon and Seimchan demonstrated the presence of two components related to the M1 and M2 sites in olivine as well as a new small component which was related to distorted M1 sites [32,33]. Moreover, a comparison of hyperfine parameters for the ${ }^{57} \mathrm{Fe}$ nuclei in the M1 and M2 sites of two olivines demonstrated small variations beyond the instrumental (systematic) error which cannot be detected in the case of low velocity resolution spectra. Using relative areas of spectral components related to the M1 and M2 sites the temperature of $\mathrm{Fe}^{2+}$ and $\mathrm{Mg}^{2+}$ equilibrium distribution in olivines was evaluated. 

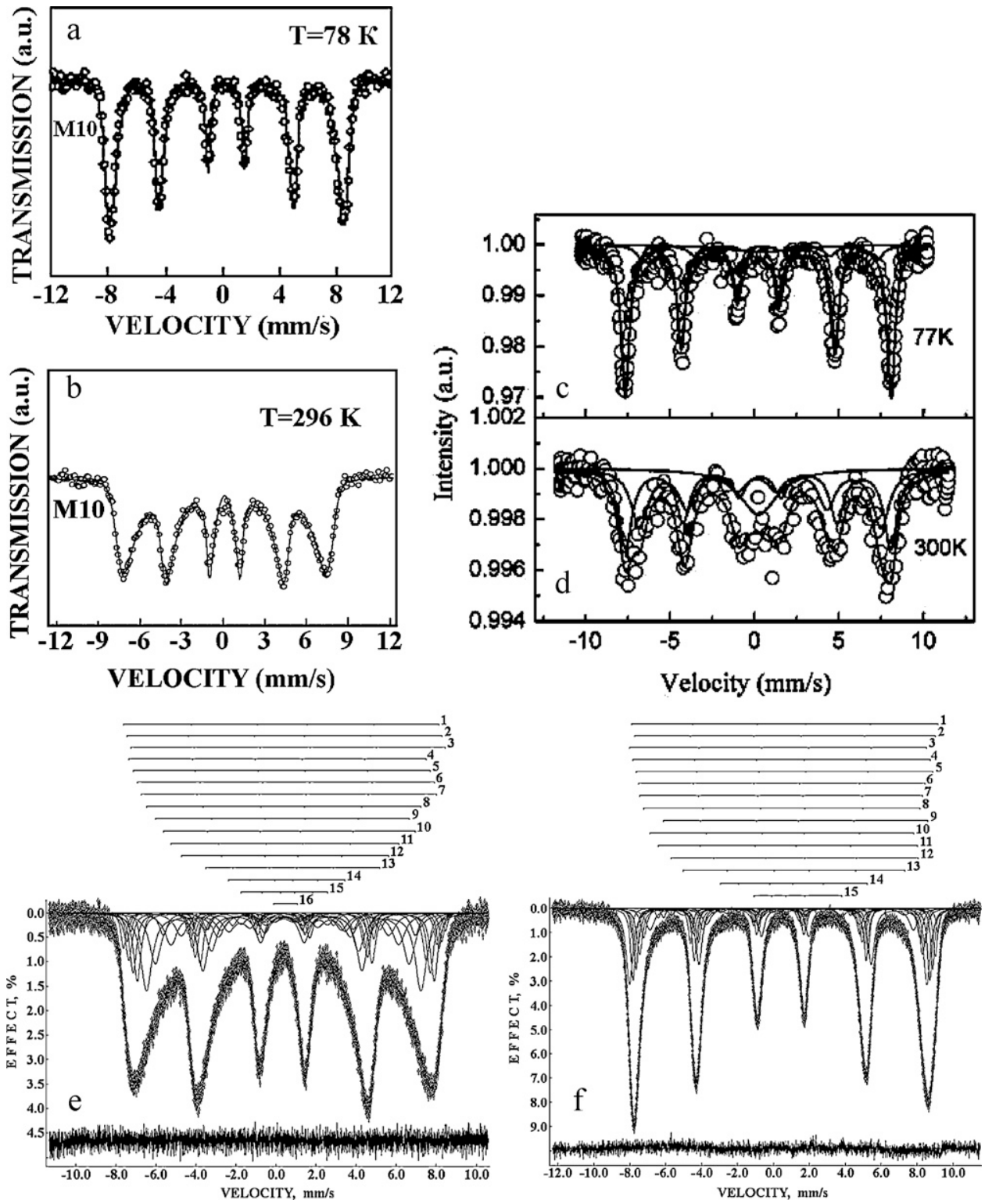

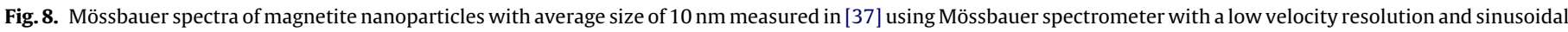

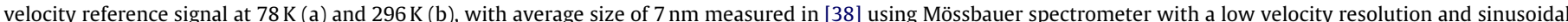

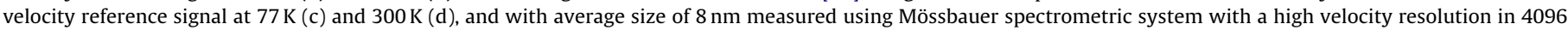
channels at $295 \mathrm{~K}$ (e) and $90 \mathrm{~K}$ (f), indicated components are the result of the best fits.

Another application of Mössbauer spectroscopy with a high velocity resolution was recently demonstrated in the study of two different morphological forms of iron nickel phosphides $(\mathrm{Fe}, \mathrm{Ni})_{3} \mathrm{P}$, schreibersite (macroinclusions) and rhabdite (microcrystals), extracted from the iron meteorite Sikhote-Alin [34-36]. Mössbauer spectra of schreibersite measured at 295 and $90 \mathrm{~K}$ with a high velocity resolution and presented in 1024 channels were fitted using 6 magnetic sextets two pairs of which were related to the ${ }^{57} \mathrm{Fe}$ in each crystallographically non-equivalent site, respectively (M1, M2 and M3). Mössbauer spectra of rhabdite were measured with a high velocity resolution at 295,220,150 and $90 \mathrm{~K}$ and demonstrated superparamagnetic behavior due to a small size of the crystals. These spectra were also fitted using 6 magnetic sextets two pairs of which were related to the ${ }^{57} \mathrm{Fe}$ in each M1, M2 and M3 site, respectively, and one quadrupole doublet related to residual paramagnetic phase which was assigned to the part of ${ }^{57} \mathrm{Fe}$ nuclei in the M3 site. Based on the fits of Mössbauer spectra of schreibersite and rhabdite the temperature dependences of magnetic hyperfine fields related to the ${ }^{57} \mathrm{Fe}$ in the M1, M2 and M3 sites were obtained. Moreover, it was possible to evaluate the $\mathrm{Fe}$ and $\mathrm{Ni}$ occupancies of the M1, M2 and M3 sites in both iron nickel phosphides which appeared to be different for schreibersite and rhabdite.

\subsection{Nanotechnological application}

A lot of nanomaterials used in various applied fields or developed for any other applications contain Fe. Therefore, new features of nanosized matter are being studied by Mössbauer spectroscopy. Some iron oxide nanoparticles are used or developed for magnetic fluids for biomedical aims. A comparison of Mössbauer spectra of magnetite $\mathrm{Fe}_{3} \mathrm{O}_{4}$ nanoparticles with the average size of 10 and $7 \mathrm{~nm}$ measured in $[37,38]$ with a low velocity resolution and of $8 \mathrm{~nm}$ measured with a high velocity resolution (our results $[39,40]$ ) are shown in Fig. 8. Spectra measured at room and liquid nitrogen temperatures are respectively similar, however, the quality of Mössbauer spectra measured with a low and high velocity resolution is 

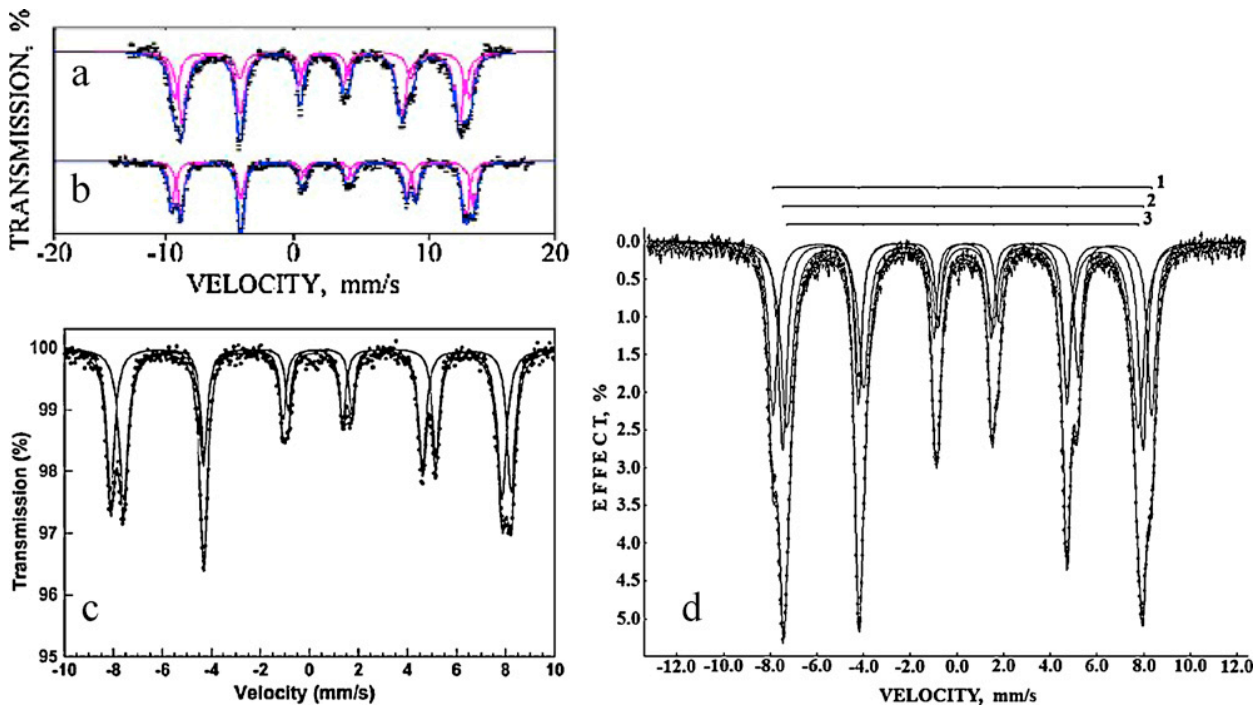

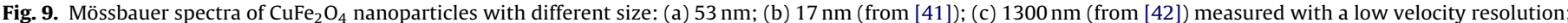
and (d) $10 \mathrm{~nm}$ measured with a high velocity resolution and presented in 1024 channels (components 1,2 and 3 are the result of the best fit); $T=295 \mathrm{~K}$.

substantially different. Therefore, Mössbauer spectra measured with a low velocity resolution were fitted using distribution of hyperfine field mainly while the high velocity resolution spectra were fitted using individual components. It should be noted that the low velocity resolution Mössbauer spectra of $\mathrm{Fe}_{3} \mathrm{O}_{4}$ nanoparticles were usually fitted using a physical model based on the spinel structure: two iron sites, tetrahedral and octahedral (two magnetic sextets mainly). In contrast, Mössbauer spectra of $\mathrm{Fe}_{3} \mathrm{O}_{4}$ nanoparticles measured with a high velocity resolution at 295 and $90 \mathrm{~K}$ cannot be fitted well using this model. The best fit of these spectra demonstrated a large number of magnetic sextets which cannot be explained now. Nevertheless, this result demonstrates that the fit of the high quality Mössbauer spectra measured with a high velocity resolution required a new physical model.

Other spinel nanoparticles such as $\mathrm{CuFe}_{2} \mathrm{O}_{4}$ are developed for anode materials in Li-ions batteries. Mössbauer spectra of some $\mathrm{CuFe}_{2} \mathrm{O}_{4}$ nanoparticles with different size $(53,17,1300$ and $10 \mathrm{~nm})$ and preparation procedure measured with a low and high velocity resolution at room temperature in [41-43] are shown in Fig. 9. In spite of different shape of the spectra the difference in the spectra quality is clearly seen. All Mössbauer spectra of $\mathrm{CuFe}_{2} \mathrm{O}_{4}$ nanoparticles measured with a low velocity resolution and spectra folding were fitted using two sextets within a model accounting for tetrahedral and octahedral sites for Fe ions. However, the high velocity resolution Mössbauer spectrum of $\mathrm{CuFe}_{2} \mathrm{O}_{4}$ nanoparticles was better fitted using 3 magnetic sextets. This fact may be a result of better sensitivity of the high velocity resolution spectra to structural peculiarities of nanoparticles which cannot be revealed from the low velocity resolution spectra.

\section{Conclusion}

One of the ways of further development of Mössbauer spectroscopy is related to increase of the spectra quality and possibility to extract more detailed information about the studied iron containing biomolecules, molecular systems and materials. In this case velocity resolution in Mössbauer spectrometer driving system and in Mössbauer spectrum should be taken into account in addition to other characteristics of both the spectrometer and the spectrum. An approach to evaluate the velocity resolution in the Doppler modulation system and in Mössbauer spectrum as well as the instrumental (systematic) error for velocity scale in the spectrum was considered. A new precision and stable Mössbauer spectrometric system created on the basis of spectrometer SM-2201 with a high velocity resolution ( 4096 bits) and liquid nitrogen cryostat with moving absorber was compared with some other Mössbauer spectrometers with a low velocity resolution (up to 1024 bits for triangular mode). It was shown that Mössbauer spectra measured with a high velocity resolution demonstrated much better quality and Lorentzian line shape. Recent results in the application of this technique for studying biological molecules such as ferritin, liver and spleen tissues, oxyhemoglobins in red blood cells from different sources including patients' blood as well as ferritin pharmaceutically important models demonstrated possibility to distinguish different biomolecules of the same type. Small variations of Mössbauer hyperfine parameters were found in vitamins and dietary supplements containing ferrous fumarate and ferrous sulfate as well as some iron containing impurities were observed in Mössbauer spectra. Mössbauer spectra of meteorite samples and various nanoparticles demonstrated higher quality spectra in comparison with those measured using the low velocity resolution spectrometers. Moreover, these spectra required new models for the best fit and, therefore, for instance, new physical models for spinel nanoparticles. Finally, we should point out both problems and advantages of the application of Mössbauer spectroscopy with a high velocity resolution based on our experience.

The main problems are as follows:

- Availability of Mössbauer spectrometer with a high velocity resolution, precision and stable velocity driving system;

- Substantially longer measurement time to reach the same signalto-noise ratio in Mössbauer spectra measured with a high velocity resolution in comparison with those measured with a low velocity resolution;

- The necessity of accounting for parabolic distortion of the spectrum base line in the case of saw-tooth shape velocity reference signal and geometry with moving source (for instance, in the case of liquid helium cryostat usage);

- The necessity of new mathematical approach in the least square method to fit Mössbauer spectra with a high velocity resolution consisted of too many components and, therefore, varied parameters. 
However, application of Mössbauer spectroscopy with a high velocity resolution demonstrated some important advantages such as:

- The fine adjustment to resonance;

- An increase in velocity resolution in Mössbauer spectroscopy leads to decrease in the instrumental (systematic) errors on the velocity scale for each spectrum point and for hyperfine parameters with much better distinguishing of small variations of these parameters;

- The fit of complicated Mössbauer spectra measured with a high velocity resolution and sufficient signal-to-noise ratio is more reliable and demonstrates more exact results;

- An increase in statistical counting rate for the complicated low velocity resolution Mössbauer spectrum cannot compensate an increase in velocity resolution in this spectrum with less statistical counting rate for more reliable fit.

Thus, application of Mössbauer spectroscopy with a high velocity resolution demonstrated new possibilities of a higher quality study of iron-containing materials especially in biomedical research and should be used for further development of investigations in this field.

\section{Acknowledgments}

We would like to thank Dr. V. Šepelák, Prof. E. Kuzmann, Dr. Sc. V.A. Shabashov and Prof. K. Ruebenbauer for their help with measurement of Mössbauer spectra of $\alpha$-Fe foils using their spectrometers. We further wish to thank our colleagues Prof. P.C. Morais, Dr. S. Kundu, Dr. R.K. Selvan, Prof. S.M. Dubiel, Dr. V. Šepelák, Dr. V.I. Grokhovsky, Dr. A.L. Berkovsky, Dr. A.V.Vinogradov, Dr. T.S. Konstantinova, Prof. N.V. Sadovnikov, Dr. E.V. Petrova, Dr. M.Yu. Larionov, E.N. Novikov, I.V. Alenkina, M.V. Ushakov and Yu.V. Klepova for continuous collaboration. We are grateful to Prof. E. Kuzmann and Prof. D.L. Nagy for stimulating discussions of methodological aspects. This work was supported in part by the Russian Foundation for Basic Research (grant \# 09-02-00055-a).

\section{References}

[1] H. Frauenfelder, The Mössbauer Effect, W.A. Benjamin, New York, 1963.

[2] V.I. Goldaskii, R.H. Herber, Chemical Applications of Mössbauer Spectroscopy, Academic Press, New York/London, 1968.

[3] N.N. Greenwood, T.C. Gibb, Mössbauer Spectroscopy, Chapman and Hall, London, 1971.

[4] P. Gutlich, R. Link, A.M. Trautwein, Mössbauer Spectroscopy and Transition Metal Chemistry, Springer-Verlag, Berlin/Heidelberg/New York, 1978.

[5] A. Vértes, L. Korecz, K.M. Burger, Mössbauer Spectroscopy, Academia Kiada, Budapest, 1979

[6] T.E. Cranshaw, B.W. Dale, C.O. Longworth, C.E. Johnson, Mössbauer Spectroscopy and its Applications, Cambridge University Press, 1985.

[7] V.A. Semionkin, S.M. Irkaev, O.B. Milder, M.I. Oshtrakh, Mössbauer Effect Ref. Data J. 28 (2005) 288-291.

[8] M.I. Oshtrakh, V.A. Semionkin, O.B. Milder, E.G. Novikov, J. Radioanal. Nucl. Chem. 281 (2009) 63-67.

[9] V.A. Semionkin, M.I. Oshtrakh, O.B. Milder, E.G. Novikov, Bull. Russ. Acad. Sci.: Phys. 74 (2010) 416-420.
[10] R. Gornicki, A. Błachowski, K. Ruebenbauer, Nukleonika 52 (2007) S7-S12.

[11] M.I. Oshtrakh, V.A. Semionkin, O.B. Milder, E.G. Novikov, Proceedings of the international conference Mössbauer spectroscopy in materials science 2008 in: M. Mashlan, R. Zboril (Eds.), AIP Conference Proceedings, Melville, New York, vol. 1070, 2008, pp. 122-130.

[12] M.I. Oshtrakh, V.A. Semionkin, V.I. Grokhovsky, O.B. Milder, E.G. Novikov, J Radioanal. Nucl. Chem. 279 (2009) 833-846.

[13] M.I. Oshtrakh, V.A. Semionkin, O.B. Milder, E.G. Novikov, J. Mol. Struct. 924-926 (2009) 20-26.

[14] M.I. Oshtrakh, V.A. Semionkin, O.B. Milder, E.G. Novikov, Bull. Russ. Acad. Sci.: Phys. 74 (2010) 407-411.

[15] M.I. Oshtrakh, V.A. Semionkin, O.B. Milder, I.V. Alenkina, E.G. Novikov, Spectroscopy 24 (2010) 593-599.

[16] M.I. Oshtrakh, I.V. Alenkina, O.B. Milder, V.A. Semionkin, Spectrochim. Acta A: Mol. Biomol. Spectrosc. 79 (2011) 777-783.

[17] M.I. Oshtrakh, O.B. Milder, V.A. Semionkin, Hyperfine Interact. 185 (2008) 39-46.

[18] M.I. Oshtrakh, A.L. Berkovsky, A. Kumar, S. Kundu, A.V. Vinogradov, T.S. Konstantinova, V.A. Semionkin, Hyperfine Interact. 197 (2010) 301-307.

[19] M.I. Oshtrakh, A. Kumar, S. Kundu, A.L. Berkovsky, V.A. Semionkin, J. Mol. Struct 993 (2011) 292-296

[20] M.I. Oshtrakh, A.L. Berkovsky, A. Kumar, S. Kundu, A.V. Vinogradov, T.S. Konstantinova, V.A. Semionkin, Biometals 24 (2011) 501-512.

[21] B. Shaanan, J. Mol. Biol. 171 (1983) 31-59.

[22] M.I. Oshtrakh, V.A. Semionkin, Hyperfine Interact. 159 (2004) 345-350.

[23] I.V. Alenkina, M.I. Oshtrakh, Yu.V. Klepova, S.M. Dubiel, N.V. Sadovnikov, V.A. Semionkin, Spectrochim. Acta A: Mol. Biomol. Spectrosc. 100 (2013) 88-93.

[24] M.I. Oshtrakh, I.V. Alenkina, S.M. Dubiel, V.A. Semionkin, J. Mol. Struct. 993 (2011) 287-291.

[25] M.I. Oshtrakh, E.G. Novikov, S.M. Dubiel, V.A. Semionkin, Proceedings of the international conference Mössbauer spectroscopy in materials science 2010 in: J. Tuček, M. Miglierini (Eds.), AIP Conference Proceedings, Melville, New York, vol. 1258, 2010, pp. 75-81.

[26] M.I. Oshtrakh, E.G. Novikov, S.M. Dubiel, V.A. Semionkin, Hyperfine Interact. 197 (2010) 287-294.

[27] V.I. Grokhovsky, E.V. Zhiganova, M.Yu. Larionov, K.A. Uimina, M.I. Oshtrakh, Phys. Metals Metallogr. 105 (2008) 177-187.

[28] M.I. Oshtrakh, E.V. Petrova, V.I. Grokhovsky, V.A. Semionkin, Meteorit. Planet Sci. 43 (2008) 941-958.

[29] M.I. Oshtrakh, V.I. Grokhovsky, E.V. Petrova, M.Yu. Larionov, K.A. Uymina, V.A Semionkin, N.V. Abramova, Proceedings of the international conference Mössbauer spectroscopy in materials science 2008, in: M. Mashlan, R. Zboril (Eds.) AIP Conference Proceedings, Melville, New York, vol. 1070, 2008, pp. 131-139.

[30] V.I. Grokhovsky, M.I. Oshtrakh, E.V. Petrova, M.Yu. Larionov, K.A. Uymina, V.A Semionkin, Eur. J. Mineral. 21 (2009) 51-63.

[31] H.C. Verma, K. Jee, R.P. Tripathi, Meteorit. Planet. Sci. 38 (2003) 963-967.

[32] D.G. Patrusheva, M.I. Oshtrakh, E.V. Petrova, V.I. Grokhovsky, V.A. Semionkin, Hyperfine Interact. 197 (2010) 295-300.

[33] M.I. Oshtrakh, D.G. Patrusheva, E.V. Petrova, V.I. Grokhovsky, V.A. Semionkin Meteorit. Planet. Sci. (Suppl.) 45 (2010) A158.

[34] M.I. Oshtrakh, M.Yu. Larionov, V.I. Grokhovsky, V.A. Semionkin, J. Alloys Compd. 509 (2011) 1781-1784

[35] M.I. Oshtrakh, M.Yu. Larionov, V.I. Grokhovsky, V.A. Semionkin, J. Mol. Struct. 993 (2011) 38-42.

[36] M.I. Oshtrakh, M.Yu. Larionov, V.I. Grokhovsky, V.A. Semionkin, Mater. Chem. Phys. 130 (2011) 373-380.

[37] G.F. Goya, T.S. Berquo, F.C. Fonseca, M.P. Morales, J. Appl. Phys. 94 (2003) 3520-3528.

[38] J.G. Santos, L.B. Silveira, Q.S. Ferreira, V.K. Garg, A.C. Oliveira, M.S. Parise, P.C Morais, J. Phys.: Conf. Ser. 214 (2010) 012133.

[39] M.I. Oshtrakh, A.F.R. Rodriguez, V.A. Semionkin, J.G. Santos, O.B. Milder, L.B. Silveira, E.M. Marmolejo, M.V.Ushakov, M. de Souza-Parise, P.C. Morais, J. Phys.: Conf. Ser. 217 (2010) 012018.

[40] M.I. Oshtrakh, V. Šepelák, A.F.R. Rodriguez, V.A. Semionkin, M.V. Ushakov, J.G. Santos, L.B. Silveira, E.M. Marmolejo, M. de Souza-Parise, P.C. Morais, Spectrochim. Acta A: Mol. Biomol. Spectrosc 100 (2013) 94-100.

[41] M.J. Iqbal, N. Yaqub, B. Sepiol, B. Ismail, Mater. Res. Bull. 46 (2011) 1837-1842.

[42] I. Nedkov, R.E. Vandenberghe, Ts. Marinova, Ph. Thailhades, T. Merodiiska, I. Avramova, Appl. Surf. Sci. 253 (2006) 2589-2596.

[43] M.I. Oshtrakh, R. Kalai Selvan, C.O. Augustin, V.A. Semionkin, Hyperfine Interact 183 (2008), 37-34. 